\title{
Consumo de bebidas alcoólicas por estudantes de escolas públicas da cidade do Recife-PE
}

Jael Maria de Aquino ${ }^{1}$

Kenned da Silva Teixeira ${ }^{1}$

Darine Marie Rodrigues da Silva ${ }^{1}$

Raquel de Figueiredo Xavier ${ }^{1}$

Silvia Elizabeth Gomes de Medeiros ${ }^{1}$

Viviane Tannuri Ferreira Lima Falcão ${ }^{1}$

\footnotetext{
Universidade de Pernambuco, Faculdade de Enfermagem Nossa Senhora das Graças, Recife, PE, Brasil.
}

Objetivo: analisar o consumo de álcool entre adolescentes das escolas públicas na cidade do Recife-PE. Método: tratase de uma pesquissa descritiva, exploratória de abordagem quantitativa, desenvolvida em três escolas públicas estaduais da cidade do Recife-PE, com a população composta por escolares de ambos os sexos, na faixa etária entre 12 a 17 anos. Resultados: o estudo identificou fatores de risco que influenciam o consumo de álcool entre os escolares entrevistados, destacando-se as variáveis idade, reprovação escolar e número de pessoas com quem mora. Conclusão: sugere-se que haja o fortalecimento de práticas de educação em saúde através de parcerias entre escola e Unidade de Saúde da Família local por meio do Programa de Saúde na Escola (PSE), que estimulem práticas favoráveis à saúde.

Descritores: Enfermagem; Consumo de Bebidas Alcoólicas; Adolescentes; Serviços de Saúde Escolar.

\section{Como citar este artigo}

Aquino JM, Teixeira KS, Silva DMR, Xavier RF, Medeiros SEG, Falcão VTFL. Consumption of alcoholic beverages by students of public schools in the city of Recife-PE. SMAD, Rev Eletrônica Saúde Mental Álcool Drog. 2019;15(2):60-68. doi: https://dx.doi.org/10.11606/issn.1806-6976.smad.2019.000419 


\section{Consumption of alcoholic beverages by students of public schools in the city of Recife-PE}

Objective: to analyze alcohol consumption among adolescents of public schools in the city of Recife-PE. Method: it is a descriptive, exploratory, quantitative approach, developed in three state public schools in the city of Recife-PE, with the population composed of schoolchildren of both sexes, between 12 and 17 years of age. Results: the study identified risk factors that influence alcohol consumption among schoolchildren interviewed, highlighting the variables age, school disapproval and number of people with whom. Conclusion: it is suggested that there be strengthened health education practices through partnerships between the school and the local Family Health Unit through the School Health Program (PSE), which stimulate health-friendly practices.

Descriptors: Nursing; Consumption of Alcoholic Beverages; Adolescents; School Health Services.

\section{Consumo de bebidas alcohólicas por estudiantes de escuelas públicas de la ciudad de Recife-PE}

Objetivo: analizar el consumo de alcohol entre adolescentes de las escuelas públicas en la ciudad de Recife-PE. Método: se trata de una pesquisa descriptiva, exploratoria de abordaje cuantitativo, desarrollada en tres escuelas públicas estatales de la ciudad de Recife-PE, con la población compuesta por escolares de ambos sexos, en el grupo de edad entre 12 a 17 años. Resultados: el estudio identificó factores de riesgo que influencian el consumo de alcohol entre los escolares entrevistados, destacándose las variables edad, reprobación escolar y número de personas con quienes. Conclusión: se sugiere que haya el fortalecimiento de prácticas de educación en salud a través de alianzas entre escuela y Unidad de Salud de la Familia local a través del Programa de Salud en la Escuela (PSE), que estimulan prácticas favorables a la salud.

Descriptores: Enfermería, Consumo de bebidas alcohólicas, Adolescentes, Servicios de salud escolar. 


\section{Introdução}

A adolescência é caracterizada por período de intensas mudanças psicossociais e biológicas, com uma maior busca por novas experiências e curiosidade por novas sensações, tendo o consumo de álcool como prática comum, nessa faixa etária, apesar da venda de bebidas alcoólicas ser proibida para menores de 18 anos $^{(1)}$.

É nesse contexto que se inserem grandes preocupações associadas a essa fase da vida, que são os riscos relacionados ao consumo de álcool e outras drogas. Os danos decorrentes do consumo de álcool em adolescentes diferem dos observados em indivíduos adultos, seja devido a especificidades psicossociais existentes nesse ciclo da vida, seja por questões neurológicas específicas em função do amadurecimento cerebral|(1).

A exacerbação do uso de álcool por adolescentes pode trazer efeitos graves à memória, inteligência, aprendizagem, além de elevar a vulnerabilidade do indivíduo ao alcoolismo devido às modificações neurofisiológicas importantes. Alguns efeitos negativos provocados pelo uso do álcool a nível emocional e cognitivo podem acompanhar o indivíduo durante a vida(2).

Segundo Garcia et al. (2015)(3), o álcool é fator causal em mais de 60 tipos de doenças e lesões e causa principal de algumas doenças, como também é um componente importante em diversas causas de óbito. Segundo o autor, de 2010 a 2012, foram registradas, no Brasil, a cada ano, quase 20 mil mortes nas quais o consumo de álcool foi condição necessária para sua ocorrência, equivalendo a mais de 1500 mortes por mês ou 50 por dia, sendo as doenças do fígado uma das principais causas de morte.

De acordo com dados da Pesquisa Nacional de

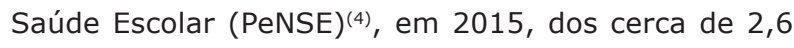
milhões de estudantes que cursavam o $9^{\circ}$ ano do ensino fundamental, 55,5\% já haviam consumido uma dose de bebida alcoólica alguma vez, percentual superior ao observado em 2012 (50,3\%). A proporção dos que já experimentaram drogas ilícitas subiu de 7,3\% para 9,0\% no mesmo período. Em relação ao consumo atual de álcool e drogas ilícitas, respectivamente, 23,8\% e 4,2\% dos estudantes tinham feito uso dessas substâncias nos últimos 30 dias antes da pesquisa.

Considerando que, além de consequências para a saúde, o uso prejudicial do álcool inflige perdas sociais e econômicas significativas sobre os indivíduos e a sociedade em geral. O uso nocivo de álcool continua a ser um fator que deve ser considerado para garantir ações sociais e desenvolvimento econômico em todo o mundo. Para a OMS, a prevenção e redução do uso nocivo do álcool devem ser tratadas como prioridade para que seja possível alcançar a meta previamente estipulada de redução relativa de $10 \%$ no consumo nocivo mundial em 2025(5).
O Relatório Mundial sobre Álcool e Saúde 2014 apresenta uma perspectiva abrangente sobre o consumo global, regional e nacional de álcool, padrões de consumo, consequências para a saúde e as respostas políticas dos países. É acima de tudo um esforço contínuo da Organização Mundial de Saúde (OMS) para recolher de informação a fim de ajudá-los em seus esforços para reduzir o uso nocivo do álcool e suas consequências à saúde ${ }^{(5)}$.

O uso de bebidas alcoólicas na adolescência é visto como um problema mundial de saúde pública, uma vez que pode trazer consequências físicas e mentais imediatas duradouras. Estando sob efeito de determinados níveis de álcool o indivíduo pode também se expor a situações de risco, como as doenças sexualmente transmissíveis, envolver-se em brigas e também em atos de vandalismo(6).

Os adolescentes fazem parte de um grupo populacional vulnerável, uma vez que necessitam de um olhar diferenciado. Os dados mostram o impacto negativo do uso regular de álcool e outras drogas nessa faixa etária. Logo, sabe-se que quanto mais precoce o início do consumo, maior o risco de surgirem consequências graves.

O conhecimento dos fatores de risco de transtornos do uso de álcool é essencial para orientar as políticas de saúde pública de vigilância, controle de riscos e danos e de promoção da saúde contra o uso abusivo de álcool, articulando-as com o envolvimento dos diversos atores, como o poder público, os educadores, a família e a sociedade em geral, visando aperfeiçoar as políticas públicas existentes, desde a regulação da oferta até a venda(7).

O presente estudo teve por objetivo analisar o consumo de álcool entre adolescentes das escolas públicas na cidade do Recife/PE e identificar as relações existentes entre o uso de álcool e os fatores de risco à saúde dos adolescentes, bem como seu desempenho escolar.

\section{Método}

Trata-se de uma pesquisa descritiva exploratória, com abordagem quantitativa, desenvolvida em três escolas estaduais da cidade do Recife/PE. Os critérios de elegibilidade foram: escolares de ambos os sexos, incluídos na faixa etária entre 12 e 17 anos, regularmente matriculados no $7^{\circ}, 8^{\circ}$ e $9^{\circ}$ anos do ensino fundamental e $1^{\circ}, 2^{\circ}$ e $3^{\circ}$ anos do ensino médio de três escolas públicas estaduais de um Distrito Sanitário da cidade do Recife. Foi critério de exclusão: discentes afastados por atestado médico ou determinações escolares. As escolas foram devidamente selecionadas através de um sorteio. Os estudantes foram selecionados de forma aleatória, de modo que todos foram convidados e compuseram a amostra aqueles que trouxeram o Termo de Consentimento assinado pelos pais ou responsável legal nos dias de coleta de dados. 
Para a determinação do tamanho amostral, utilizou-se a equação de cálculo de amostra para estudo de proporção em população finita, dada por: $\mathrm{n}=\frac{z^{2} p q N}{d^{2}(N-1)+z^{2} p q}$, em que $z$ = quantil da normal padrão $(1,96$, quando considerado um coeficiente de confiança de $95 \%$ ); $p$ = proporção esperada de alunos com risco para dependência de bebida alcoólica $(p=0,1) ; q=$ proporção esperada de alunos sem risco para dependência de bebida alcoólica ( $q=1-0,1=0,9)$; $\mathrm{d}=$ erro amostral $(\mathrm{d}=0,05) ; \mathrm{N}=$ Número total de alunos matriculados nas escolas avaliadas no ano de 2015 ( $\mathrm{N}=$ 2909), considerando um nível de significância de 95\%, prevalência esperada de dependência alcoólica de 10\%, erro amostral de 5\% e o número total de 2909 alunos matriculados nas escolas em estudo, sendo a amostra constituída por 132 alunos.

Para coleta de dados foi utilizado um questionário com questões sociodemográficas, elaborado pelos autores, e outro instrumento, o Alcohol Use Disorders Indentification Test - AUDIT, desenvolvido pela Organização Mundial da Saúde (OMS), que teve por objetivo identificar a ingestão prejudicial e potencialmente perigosa do álcool.

O formulário sociodemográfico é composto por variáveis referentes à idade, sexo, raça, escolaridade, ocupação, religião, escolaridade dos pais, estado civil dos pais e renda familiar. Já o AUDIT é composto por 10 itens, cada um com margem de 0 a 4 pontos, possibilitando um espectro de pontuação de 0 a 40 . A pontuação que o sujeito atinge ao responder aos itens do AUDIT permite a classificação do uso da substância da seguinte forma: Zona I (baixo risco) - 0 a 7 pontos; Zona II (uso de risco) - 8 a 15 pontos; Zona III (uso nocivo) - 16 a 19 pontos; Zona IV (provável dependência) - 20 a 40 pontos $^{(8)}$.

A coleta dos dados ocorreu no período de julho a novembro de 2015 e foi organizada da seguinte maneira: em um primeiro momento, houve um contato com a direção das escolas onde foram explicados os objetivos da pesquisa e a sua finalidade. Nesse momento foi obtida a autorização para visitar o ambiente escolar e manter aproximação com os estudantes. Em um segundo momento foram explicados os objetivos da pesquisa e a importância da participação da mesma aos estudantes. Então, foram convidados a participar, distribuídos os termos de consentimento e solicitada a assinatura desse termo pelos pais ou responsável legal. Posteriormente, realizou-se a coleta dos dados com aqueles estudantes que aceitaram participar da pesquisa e que trouxeram, no momento, o termo de consentimento assinado pelos pais ou responsável legal.

Os dados foram analisados pelo software SPSS, versão 17. Na análise dos dados sociodemográficos foram calculadas as frequências absolutas e relativas e para avaliar o grau de dependência dos alunos ao uso do álcool foi calculado o escore de dependência de cada aluno com base no AUDIT, sendo realizada a análise descritiva do escore através das estatísticas: mínimo, máximo, média, desvio padrão e Teste Exato de Fisher. A normalidade do escore do AUDIT utilizou o teste de Mann-whitney para comparar a distribuição do escore entre dois grupos avaliados e o teste de Kruskall-wallis na comparação entre mais de três grupos. Todas as conclusões foram tiradas considerando o nível de significância de 5\%.

O desenvolvimento da pesquisa implicou na observância da Resolução no. 466/12 do Ministério da Saúde que norteia a pesquisa envolvendo seres humanos. O projeto foi aprovado pelo Comitê de Ética em Pesquisa do Complexo com o Parecer n 1.169.254.

\section{Resultados}

Conforme demonstra a Tabela 1, a amostra foi composta por 132 (100\%) estudantes, dos quais a maioria era do sexo feminino $(63,6 \%)$, tinha idade igual a 15 anos (25\%), declarou-se da raça parda (48,5\%), era residente da zona urbana $(93,7 \%)$, seguia a religião evangélica $(46,2 \%)$ e não trabalhava $(92,4 \%)$.

Tabela 1 - Distribuição do perfil pessoal dos estudantes de escolas públicas da cidade do Recife/PE, Brasil, 2015

\begin{tabular}{lcc}
\hline Fator avaliado & N & $\%$ \\
\hline Q2 - Sexo & & \\
Masculino & 48 & 36,4 \\
Feminino & 84 & 63,6 \\
Q1 - Idade & & \\
12 & 14 & 10,5 \\
13 & 19 & 14,4 \\
14 & 27 & 20,5 \\
15 & 33 & 25,0 \\
16 & 17 & 12,9 \\
17 & 22 & 16,7 \\
Q3 - Local de residência & & \\
Zona urbana & 119 & 93,7 \\
Zona rural & 8 & 6,3 \\
Q6 - Religião & & \\
Católico & 33 & 25,0 \\
Evangélico & 61 & 46,2 \\
Espírita & 7 & 5,3 \\
$\quad$ Não tem religião & 31 & 23,5 \\
Q7 - Raça & & \\
Branco & 30 & 22,7 \\
Pardo & 64 & 48,5 \\
Negro & 30 & 22,7 \\
Amarelo & 8 & 6,1 \\
Q8 - Trabalha & & \\
Sim & 10 & 7,6 \\
Não & 121 & 92,4 \\
\hline
\end{tabular}

Segundo a composição familiar, destacaram-se os seguintes resultados: $56,9 \%$ dos participantes moravam com pai/mãe, 60,9\% residiam com 1 a 3 pessoas, $47,3 \%$ tinham pais casados, $54,9 \%$ dos pais tinham ensino médio completo/incompleto, $45,4 \%$ das mães tinham ensino médio completo/incompleto e $62,1 \%$ apresentavam renda maior que um salário mínimo, conforme expressa a Tabela 2. 
Tabela 2 - Distribuição do perfil familiar dos estudantes de escolas públicas da cidade do Recife/PE, Brasil, 2015

\begin{tabular}{|c|c|c|}
\hline Fator avaliado & $\mathbf{N}$ & $\%$ \\
\hline \multicolumn{3}{|l|}{ Q9 - Com quem mora } \\
\hline Somente pai com ou sem irmãos/avós & 3 & 2,3 \\
\hline Somente mãe com ou sem irmãos/avós & 39 & 29,5 \\
\hline Pai e mãe com ou sem irmãs/avós & 75 & 56,9 \\
\hline Apenas com os avós & 13 & 9,8 \\
\hline Outros & 2 & 1,5 \\
\hline \multicolumn{3}{|l|}{ Q10 - Quantas pessoas moram com você } \\
\hline 1 a 3 pessoas & 78 & 60,9 \\
\hline 4 a 5 pessoas & 40 & 31,3 \\
\hline Mais de 5 pessoas & 10 & 7,8 \\
\hline \multicolumn{3}{|l|}{ Q11 - Estado civil dos pais } \\
\hline Solteiros & 49 & 37,4 \\
\hline Casados & 62 & 47,3 \\
\hline Divorciados & 19 & 14,5 \\
\hline Viúvo & 1 & 0,8 \\
\hline \multicolumn{3}{|l|}{ Q12 - Renda familiar } \\
\hline Menos de 1 SM & 15 & 12,1 \\
\hline $1 \mathrm{SM}$ & 32 & 25,8 \\
\hline Mais de $1 \mathrm{SM}$ & 77 & 62,1 \\
\hline \multicolumn{3}{|l|}{ Q13 - Escolaridade do pai } \\
\hline Analfabeto & 3 & 2,4 \\
\hline Fundamental completo/incompleto & 39 & 32,0 \\
\hline Médio completo/incompleto & 67 & 54,9 \\
\hline Superior completo/incompleto & 13 & 10,7 \\
\hline \multicolumn{3}{|l|}{ Q14 - Escolaridade da mãe } \\
\hline Analfabeto & 1 & 0,8 \\
\hline Fundamental completo/incompleto & 48 & 36,9 \\
\hline Médio completo/incompleto & 59 & 45,4 \\
\hline Superior completo/incompleto & 22 & 16,9 \\
\hline
\end{tabular}

Com relação ao perfil escolar dos alunos avaliados, verifica-se que $50,8 \%$ pertencem ao ensino fundamental, em que $29,6 \%$ dos entrevistados cursavam o $1^{\circ}$ ano do ensino médio e $20,6 \%$ dos alunos refeririam já ter experiência de reprovação escolar (Tabela 3).

Na Tabela 4, tem-se a distribuição da classificação e análise descritiva do escore de risco para o consumo de bebida alcoólica. Através dela, verifica-se que a maioria dos alunos apresentou risco moderado para bebida alcoólica (95,5\%). Em média, o grupo apresenta um escore de 1,5 com desvio padrão de 3,2. O intervalo de confiança para a média do escore do AUDIT, com $95 \%$ de confiança, foi de 0,9 a 2 pontos.
Tabela 3 - Distribuição do perfil escolar dos estudantes de escolas públicas da cidade do Recife/PE, Brasil, 2015

\begin{tabular}{lcc}
\hline Fator avaliado & $\mathbf{N}$ & $\%$ \\
\hline Q4 - Série & & \\
$7^{\circ}$ ano & 17 & 12,9 \\
$8^{\circ}$ ano & 21 & 15,9 \\
$9^{\circ}$ ano & 29 & 22,0 \\
$1^{\circ}$ ano & 39 & 29,6 \\
$2^{\circ}$ ano & 13 & 9,8 \\
$3^{\circ}$ ano & 13 & 9,8 \\
Q5 - Reprovação escolar & & \\
Sim & 27 & 20,6 \\
Não & 104 & 79,4 \\
\hline
\end{tabular}

Tabela 4 - Distribuição da classificação e análise descritiva do escore de risco para o consumo de bebida alcoólica dos estudantes de escolas públicas da cidade do Recife/PE, Brasil, 2015

\begin{tabular}{lcc}
\hline Classificação do risco & $\mathbf{N}$ & $\%$ \\
\hline Moderado (0 a 7 pontos) & 126 & 95,5 \\
Risco (8 a 15 pontos) & 4 & 3,0 \\
Alto risco (16 a 19 pontos) & 2 & 1,5 \\
Mínimo & 0 & \\
Máximo & 19 & \\
Média \pm Desvio padrão & $1,5 \pm 3,2$ & \\
IC(95\%) & $0,9-2,0$ & \\
\hline
\end{tabular}

$\mathrm{Na}$ Tabela 5, apresentam-se a distribuição da classificação e o valor da média do escore do AUDIT. Através dela, verifica-se que os seguintes fatores foram significativos na avaliação da média do escore: idade ( $p$-valor $=0,014)$, reprovação escolar ( $p$-valor $=0,041$ ) e número de pessoas com quem reside ( $p$-valor $=0,001)$. Ainda, observa-se que a maior média do escore AUDIT foi obtida no grupo de alunos com idade de 15 anos (média $=2,33$ ), que já possui experiência com reprovação (média $=2,52$ ) e que mora com mais de 5 pessoas (média $=4,90$ ). Constatou-se também a influência das variáveis renda familiar, com quem mora e gênero nos valores pontuados pelos scores do AUDIT.

Tabela 5 - Distribuição da classificação da dependência e análise descritiva do AUDIT, Recife/PE, Brasil, 2015

\begin{tabular}{|c|c|c|c|c|}
\hline \multirow{2}{*}{ Fator avaliado } & \multicolumn{3}{|c|}{ Classificação do AUDIT } & \multirow{2}{*}{ Média $\pm D F$} \\
\hline & Moderado & Padrão de risco & Alto risco & \\
\hline \multicolumn{5}{|l|}{ Q2 - Sexo } \\
\hline Masculino & $45(93,8 \%)$ & $2(4,2 \%)$ & $1(2,1 \%)$ & $1,71 \pm 3,68$ \\
\hline Feminino & $81(96,4 \%)$ & $2(2,4 \%)$ & $1(1,2 \%)$ & $1,37 \pm 2,93$ \\
\hline$p$-valor & & $0,823^{*}$ & & $0,749^{+}$ \\
\hline \multicolumn{5}{|l|}{ Q1 - Idade } \\
\hline 12 & $14(100,0 \%)$ & $0(0,0 \%)$ & $0(0,0 \%)$ & $0,36 \pm 1,08$ \\
\hline 13 & $19(100,0 \%)$ & $0(0,0 \%)$ & $0(0,0 \%)$ & $0,21 \pm 0,54$ \\
\hline 14 & $26(96,3 \%)$ & $0(0,0 \%)$ & $1(3,7 \%)$ & $1,48 \pm 3,48$ \\
\hline 15 & $30(90,9 \%)$ & $3(9,1 \%)$ & $0(0,0 \%)$ & $2,33 \pm 3,77$ \\
\hline 16 & $15(88,2 \%)$ & $1(5,9 \%)$ & $1(5,9 \%)$ & $2,12 \pm 5,00$ \\
\hline 17 & $22(100,0 \%)$ & $0(0,0 \%)$ & $0(0,0 \%)$ & $1,59 \pm 2,02$ \\
\hline p-valor & & $0,295^{*}$ & & $\mathbf{0 , 0 1 4 ^ { \ddagger }}$ \\
\hline \multicolumn{5}{|c|}{ Q3 - Local de residência } \\
\hline Zona urbana & $113(95,0 \%)$ & $4(3,4 \%)$ & $2(1,6 \%)$ & $1,52 \pm 3,34$ \\
\hline Zona rural & $8(100,0 \%)$ & $0(0,0 \%)$ & $0(0,0 \%)$ & $1,50 \pm 1,78$ \\
\hline$p$-valor & & $1,000^{*}$ & & $0,194^{+}$ \\
\hline
\end{tabular}


Tabela 5 - continuação

\begin{tabular}{|c|c|c|c|c|}
\hline \multirow{2}{*}{ Fator avaliado } & \multicolumn{3}{|c|}{ Classificação do AUDIT } & \multirow{2}{*}{ Média $\pm D P$} \\
\hline & Moderado & Padrão de risco & Alto risco & \\
\hline \multicolumn{5}{|l|}{ Q6 - Religião } \\
\hline Católico & $32(97,0 \%)$ & $0(0,0 \%)$ & $1(3,0 \%)$ & $1,61 \pm 3,11$ \\
\hline Evangélico & $57(93,4 \%)$ & $3(4,9 \%)$ & $1(1,6 \%)$ & $1,57 \pm 3,76$ \\
\hline Espírita & $7(100,0 \%)$ & $0(0,0 \%)$ & $0(0,0 \%)$ & $0,71 \pm 1,50$ \\
\hline Não tem religião & $30(96,8 \%)$ & $1(3,2 \%)$ & $0(0,0 \%)$ & $1,39 \pm 2,43$ \\
\hline$p$-valor & & $0,875^{*}$ & & $0,660^{\ddagger}$ \\
\hline \multicolumn{5}{|l|}{ Q7 - Raça/Etnia } \\
\hline Branco & $28(93,4 \%)$ & $1(3,3 \%)$ & $1(3,3 \%)$ & $1,87 \pm 3,44$ \\
\hline Pardo & $60(93,8 \%)$ & $3(4,7 \%)$ & $1(1,5 \%)$ & $1,63 \pm 3,75$ \\
\hline Negro & $30(100,0 \%)$ & $0(0,0 \%)$ & $0(0,0 \%)$ & $0,93 \pm 1,66$ \\
\hline Amarelo & $8(100,0 \%)$ & $0(0,0 \%)$ & $0(0,0 \%)$ & $1,13 \pm 2,10$ \\
\hline$p$-valor & & $0,806^{*}$ & & $0,345^{\ddagger}$ \\
\hline \multicolumn{5}{|l|}{ Q8 - Trabalha } \\
\hline Sim & $9(90,0 \%)$ & $1(10,0 \%)$ & $0(0,0 \%)$ & $1,40 \pm 2,63$ \\
\hline Não & $116(95,9 \%)$ & $3(2,5 \%)$ & $2(1,6 \%)$ & $1,50 \pm 3,28$ \\
\hline$p$-valor & & $0,385^{\star}$ & & $0,753^{+}$ \\
\hline \multicolumn{5}{|l|}{ Q4 - Série } \\
\hline $7^{\circ}$ ano & $17(100,0 \%)$ & $0(0,0 \%)$ & $0(0,0 \%)$ & $0,29 \pm 0,98$ \\
\hline $8^{\circ}$ ano & $21(100,0 \%)$ & $0(0,0 \%)$ & $0(0,0 \%)$ & $0,48 \pm 1,03$ \\
\hline $9^{\circ}$ ano & $26(89,7 \%)$ & $2(6,9 \%)$ & $1(3,4 \%)$ & $2,38 \pm 3,90$ \\
\hline $1^{\circ}$ ano & $36(92,3 \%)$ & $2(5,1 \%)$ & $1(2,6 \%)$ & $1,97 \pm 4,34$ \\
\hline $2^{\circ}$ ano & $13(100,0 \%)$ & $0(0,0 \%)$ & $0(0,0 \%)$ & $1,85 \pm 2,23$ \\
\hline $3^{\circ}$ ano & $13(100,0 \%)$ & $0(0,0 \%)$ & $0(0,0 \%)$ & $0,92 \pm 1,71$ \\
\hline$p$-valor & & $0,970^{*}$ & & $0,104^{\ddagger}$ \\
\hline \multicolumn{5}{|l|}{ Q5 - Reprovação escolar } \\
\hline Sim & $24(88,9 \%)$ & $2(7,4 \%)$ & $1(3,7 \%)$ & $2,52 \pm 4,20$ \\
\hline Não & $101(97,1 \%)$ & $2(1,9 \%)$ & $1(1,0 \%)$ & $1,21 \pm 2,88$ \\
\hline$p$-valor & & $0,119^{*}$ & & $0,041^{+}$ \\
\hline \multicolumn{5}{|l|}{ Q9 - Com quem mora } \\
\hline Somente pai com ou sem irmãos/avós & $3(100,0 \%)$ & $0(0,0 \%)$ & $0(0,0 \%)$ & $1,33 \pm 2,31$ \\
\hline Somente mãe com ou sem irmãos/avós & $36(92,3 \%)$ & $3(7,7 \%)$ & $0(0,0 \%)$ & $1,67 \pm 3,04$ \\
\hline Pai e mãe com ou sem irmãs/avós & $73(97,4 \%)$ & $1(1,3 \%)$ & $1(1,3 \%)$ & $1,17 \pm 3,06$ \\
\hline Apenas com os avós & $12(92,3 \%)$ & $0(0,0 \%)$ & $1(7,7 \%)$ & $2,62 \pm 4,59$ \\
\hline Outros & $2(100,0 \%)$ & $0(0,0 \%)$ & $0(0,0 \%)$ & $3,00 \pm 4,24$ \\
\hline$p$-valor & & $0,309^{*}$ & & $0,524^{\ddagger}$ \\
\hline \multicolumn{5}{|l|}{ Q10 - Quantas pessoas moram com você } \\
\hline 1 a 3 pessoas & $74(94,9 \%)$ & $3(3,8 \%)$ & $1(1,3 \%)$ & $1,56 \pm 3,29$ \\
\hline 4 a 5 pessoas & $40(100,0 \%)$ & $0(0,0 \%)$ & $0(0,0 \%)$ & $0,63 \pm 1,50$ \\
\hline Mais de 5 pessoas & $8(80,0 \%)$ & $1(10,0 \%)$ & $1(10,0 \%)$ & $4,90 \pm 5,57$ \\
\hline$p$-valor & & $0,0913^{*}$ & & $0,001^{\ddagger}$ \\
\hline \multicolumn{5}{|l|}{ Q11 - Estado civil dos pais } \\
\hline Solteiros & $44(89,8 \%)$ & $4(8,2 \%)$ & $1(2,0 \%)$ & $0,58 \pm 4,05$ \\
\hline Casados & $61(98,4 \%)$ & $0(0,0 \%)$ & $1(1,6 \%)$ & $0,97 \pm 2,75$ \\
\hline Divorciados & $19(100,0 \%)$ & $0(0,0 \%)$ & $0(0,0 \%)$ & $1,42 \pm 1,84$ \\
\hline Viúvo & $1(100,0 \%)$ & $0(0,0 \%)$ & $0(0,0 \%)$ & --- \\
\hline$p$-valor & & $0,158^{*}$ & & $0,228^{+}$ \\
\hline \multicolumn{5}{|l|}{ Q12 - Renda familiar } \\
\hline Menos de 1 SM & $14(93,3 \%)$ & $1(6,7 \%)$ & $0(0,0 \%)$ & $1,67 \pm 3,81$ \\
\hline $1 \mathrm{SM}$ & $32(100,0 \%)$ & $0(0,0 \%)$ & $0(0,0 \%)$ & $0,59 \pm 1,21$ \\
\hline Mais de $1 \mathrm{SM}$ & $73(94,8 \%)$ & $3(3,9 \%)$ & $1(1,3 \%)$ & $1,73 \pm 3,34$ \\
\hline$p$-valor & & $0,568^{*}$ & & $0,270^{\ddagger}$ \\
\hline \multicolumn{5}{|l|}{ Q13 - Escolaridade dos pais } \\
\hline Analfabeto & $3(100,0 \%)$ & $0(0,0 \%)$ & $0(0,0 \%)$ & ---- \\
\hline Fundamental completo/incompleto & $39(100,0 \%)$ & $0(0,0 \%)$ & $0(0,0 \%)$ & $0,64 \pm 1,51$ \\
\hline Médio completo/incompleto & $62(92,5 \%)$ & $4(6,0 \%)$ & $1(1,5 \%)$ & $1,93 \pm 3,74$ \\
\hline Superior completo/incompleto & $13(100,0 \%)$ & $0(0,0 \%)$ & $0(0,0 \%)$ & $1,38 \pm 2,22$ \\
\hline$p$-valor & & $0,585^{*}$ & & $0,205^{\ddagger}$ \\
\hline \multicolumn{5}{|l|}{ Q14 - Escolaridade da mãe } \\
\hline Analfabeto & $1(100,0 \%)$ & $0(0,0 \%)$ & $0(0,0 \%)$ & --- \\
\hline Fundamental completo/incompleto & $47(97,9 \%)$ & $1(2,1 \%)$ & $0(0,0 \%)$ & $1,15 \pm 2,62$ \\
\hline Médio completo/incompleto & $55(93,2 \%)$ & $2(3,4 \%)$ & $2(3,4 \%)$ & $1,88 \pm 3,94$ \\
\hline Superior completo/incompleto & $21(95,5 \%)$ & $1(4,5 \%)$ & $0(0,0 \%)$ & $1,41 \pm 2,28$ \\
\hline$p$-valor & & $0,765^{*}$ & & $0,728^{\ddagger}$ \\
\hline
\end{tabular}

*p-valor do teste Exato de Fisher (se p-valor $<0,05$, o fator avaliado influencia na classificação do AUDIT); †p-valor do teste de Mann-Whitney (se p-valor < 0,05, a distribuição difere entre os níveis do fator avaliado). $\neq \mathrm{p}$-valor do teste de Kruskal-Wallis (se $\mathrm{p}$-valor $<0,05$, a distribuição difere entre os níveis do fator avaliado). 


\section{Discussão}

A adolescência é a etapa de maior propensão à experimentação e consumo abusivo de bebidas alcoólicas, sendo diversos os fatores que contribuem para essa realidade ${ }^{(9)}$. O consumo de bebidas alcoólicas nessa fase é um hábito que torna maior a probabilidade deste se fazer presente na idade adulta de forma abusiva e capaz de oferecer dependência(10).

Os resultados da classificação do escore de risco demonstraram que poucos estudantes fizeram consumo de bebidas alcoólicas de risco. No que diz respeito ao padrão de consumo dos adolescentes, que tipicamente é feito de forma abusiva e com diversos danos à saúde, pode-se dizer que o grupo estudado se comportou diferente, estando menos exposto a esses malefícios ${ }^{(1)}$.

No que se refere à variável sexo, não houve diferença significativa entre os padrões de consumo. No entanto, um fator que merece destaque é o quantitativo de participantes inclusos na Zona I, onde houve a maior prevalência para o sexo feminino. Por apresentarem metabolização mais lenta ao consumirem bebidas alcoólicas, as mulheres estão mais propensas aos malefícios produzidos por essas substâncias mesmo consumindo baixa quantidade e por pouco tempo(11).

Fatores relacionados às condições fisiológicas femininas, que envolvem baixos níveis séricos de álcool desidrogenase, maior quantidade de gordura no corpo em relação à água, bem como as alterações na metabolização do álcool a depender do ciclo menstrual, podem oferecer maior probabilidade à dependência em relação aos homens. Ainda, o consumo e experimentação de bebidas alcoólicas por mulheres podem ser frutos de mudanças ocorridas, que favoreceram o acesso das mulheres a determinados locais, antes de acesso ao público masculino(12).

Associação entre a idade e o consumo de bebidas alcoólicas pôde ser constatada no presente estudo. Assim, os participantes que assumiram os maiores padrões de consumo estiveram locados entre os adolescentes que possuíam maiores valores de idade. Isso pode ser explicado pelo fato de que com o aumento da idade há uma maior probabilidade de o indivíduo assumir maiores padrões de consumo de bebidas alcoólicas ${ }^{(13)}$.

Conhecer os padrões de consumo de drogas é fundamental para a elaboração de políticas públicas que prezem pela prevenção e tratamento. Estas podem ser construídas mediante a constatação dos grupos que são mais atingidos e que apresentem determinados padrões de consumo(14).

A reprovação escolar é vista como um fator que pode anteceder ou ser resultante do consumo de drogas $^{(15)}$. Neste estudo, a associação entre reprovação escolar e o consumo de drogas foi revelada quanto ao padrão de consumo. Dessa forma, para o menor padrão de consumo, o risco moderado foi observado em sua maioria na inserção dos participantes que não apresentaram histórico de reprovação.

Um fator que chamou atenção foi a ausência da figura paterna e a alteração nos padrões de risco e alto risco segundo o questionário AUDIT. Constatou-se que o quantitativo de participantes que mora apenas com a mãe possui um padrão de risco mais elevado, enquanto que os que moram com ambos os pais estão presentes em sua maioria no menor padrão de risco, com isso não possuir a figura paterna triplicou os dados da amostra nos padrões de risco e alto risco.

Os resultados de uma pesquisa realizada em 6.837 jovens australianos entre 11 e 13 anos indicaram que a relação emocional com o genitor do sexo oposto e a ausência paterna ou materna influenciaram no comportamento do filho com relação ao consumo de álcool. Os autores deste estudo ainda trazem a associação da proximidade emocional com a figura do sexo oposto ocorrer nos períodos de transição como a entrada da puberdade, o que pode explicar a incidência do consumo entre as meninas e o maior risco quando residem apenas com a figura materna(16).

A relação entre a quantidade de pessoas com a qual o adolescente reside e o padrão de consumo de bebidas alcoólicas pôde ser evidenciada, de modo que o maior quantitativo de adolescentes que fizeram o consumo de bebidas alcoólicas de baixo risco esteve presente no grupo que residia com a menor quantidade de pessoas. Esse achado nos leva a inferir que um núcleo familiar que possui quantitativo menor de integrantes permite que os pais ou outros responsáveis melhor assistam seus filhos. Nesse sentido, é relevante que seja estimulada a realização de práticas que aumentem a coesão entre os membros da família e que os pais e ou responsáveis demonstrem interesse pela rotina e hábitos dos filhos, tendo em vista que a negligência do cuidado dos pais pode estimular o uso de substâncias psicoativas ${ }^{(17)}$.

Outro fator relevante no que diz respeito ao consumo de bebidas alcoólicas é a renda familiar, sobre este não foi constatada associação estatística significativa, porém se observa que a quantidade maior de participantes que fizeram o consumo de bebidas alcoólicas de risco esteve locada no grupo que tinha renda maior que um salário mínimo. Em relação à renda, outro estudo constatou a maior prevalência de participantes que consomem bebidas alcoólicas entre aqueles que possuem maior renda(18).

Pelo fato da adolescência ser uma fase propícia à adesão de hábitos e comportamentos que podem ser difíceis de serem modificados na fase adulta, torna-se importante criar estratégias de educação em saúde que vise à promoção da saúde e práticas de autocuidado de modo a fazer o adolescente o protagonista do 
seu cuidado. O envolvimento de atores sociais, como profissionais de saúde, professores, pais e familiares, é relevante tanto na concepção como na execução dessas estratégias, sendo a prática de educação em saúde feita em espaços abertos para diálogo e atividades que permitam aflorar a criticidade sobre o valor de estabelecer hábitos saudáveis na vida dos adolescentes ${ }^{(19)}$.

\section{Conclusão}

Através deste estudo foi possível constatar a precocidade da experimentação de álcool pelos adolescentes. Quanto ao padrão de consumo de álcool evidenciado pelo AUDIT, observou-se que mesmo em menor proporção alguns estudantes apresentaram elevados níveis de consumo, enquadrados como consumo de risco e alto risco, conferindo, assim, maior vulnerabilidade à saúde. Embora a minoria tenha apresentado esse padrão, a maioria dos estudantes consumidores foram enquadrados no padrão de risco moderado, estando estes, dessa forma, vulneráveis a padrões maiores de consumo.

Nesta pesquisa, verificou-se também que diante das associações efetuadas da média dos escores do AUDIT com as variáveis sociodemográficas trabalhadas os fatores que se mostraram significantes foram idade, reprovação escolar e número de pessoas com quem reside. No entanto, observou-se a influência das variáveis renda familiar, com quem mora e gênero nos valores pontuados pelos scores do AUDIT.

Sugere-se que sejam realizadas maiores pesquisas que envolvam a temática abordada com vistas a contribuir com o aporte científico e que haja o fortalecimento de práticas de educação em saúde que estimulem práticas favoráveis à saúde, sobretudo no que diz respeito ao consumo de substâncias psicoativas. Práticas nesse âmbito podem ser fortalecidas e construídas no meio escolar através de parcerias entre escola e Unidade de Saúde da Família local por meio do Programa de Saúde na Escola (PSE). Dessa forma, espera-se que haja a construção de novos conhecimentos e fortalecimento daqueles já adquiridos. Assim, espera-se que os adolescentes possam direcionar cada vez mais seus comportamentos e práticas a favor de sua saúde.

\section{Referências}

1. Malta DC, Machado IE, Porto DL, Silva MMA, Freitas PC, Costa AWN et al. Consumo de álcool entre adolescentes brasileiros segundo a Pesquisa Nacional de Saúde Escolar (PeNSE 2012). Rev Bras Epidemiol. [Internet] 2014;(suppl pense); 203-14 [Acesso 16 nov 2018]. Disponível em: http://www.scielo.br/pdf/rbepid/ v17s1/pt_1415-790X-rbepid-17-s1-00203.pdf
2. Pierobon M, Barak M, Hazrati S, Jacobsen KH. Consumo de álcool e violência entre adolescentes argentinos. J Pediatr. [Internet] 2013; [Acesso 27 jan 2017]; 89(1):100-7. Disponível em: http://www.scielo.br/scielo. php?script $=$ sci_arttext\&pid $=$ S0021-75572013000100015 3. Garcia LP, Freitas LRS, Gawryszewski VP, Duarte EC. Uso de álcool como causa necessária de morte no Brasil, 2010 a 2012. Rev Panam Salud Pública [Internet]. 2015 [Acesso 17 ago 2017];4(38):418-24. Disponível em: http://www.scielosp.org/pdf/rpsp/v38n5/v38n5a10.pdf. 4. Instituto brasileiro de geografia e estatística- IBGE. Pesquisa nacional de saúde do escolar 2015. Rio de Janeiro; 2016.

5. World Health Organization. Global status report on alcohol and health. Geneva; 2014. p. 392. Available from: apps.who.int/iris/ bitstream/10665/112736/1/9789240692763_eng.pdf. 6. Anjos KF, Santos VC, Almeida OS. Caracterização do consumo de álcool entre estudantes do ensino médio. Rev Baiana Saúde Pública. [Internet]. 2012; [Acesso 27 jan 2017]; 36(supl 2):418-31. Disponível em: http://inseer. ibict.br/rbsp/index.php/rbsp/article/viewFile/468/pdf_149 7. Malta DC, Mascarenhas MDM, Porto DL, Barreto SM, Neto OLM. Exposure to alcohol among adolescent students and associated factors. Reve Saúde Pública. [Internet]. 2014 [cited 2017 Sept 10]; 48(1): 52-62. Available from: http://www.scielo.br/scielo.php?script=sci_arttex t\&pid=S0034-89102014000100052.

8. World Health Organization. The Alcohol Use Disorders Identification Test. 2.ed. Geneva: 2011.

9. Elicker E, Palazzo LS, Aerts DRGC, Alves GG, Câmara S. Uso de álcool, tabaco e outras drogas por adolescentes escolares de Porto Velho-RO, Brasil. Epidemiol Serv Saúde. [Internet]. 2015; 24(3):399-410 [Acesso 16 nov 2018]. Disponível em: http://www.scielo.br/scielo. php?script=sci_arttext\&pid=S2237-96222015000300399 10. Reis TG, Oliveira LCM. Padrão de consumo de álcool e fatores associados em adolescentes estudantes de escolas públicas de um município do interior do Brasil. Rev Bras Epidemiol. [Internet] 2015;18(1):13-24 [Acesso 16 nov 2018]. Disponível em: http://www.scielo. br/scielo.php?script=sci_arttext\&pid=S1415-790X20150 00100013\&lng=en\&tIng=en

11. Dallo L, Martins RA. Associação entre as condutas de risco do uso de álcool e sexo desprotegido em adolescentes numa cidade do Sul do Brasil. Ciênc Saúde Coletiva. [Internet] 2018; 23(1):303-14. [Acesso 16 nov 2018]. Disponível em: https:// www.scielosp.org/scielo.php?script $=$ sci_arttext\&pi $\mathrm{d}=\mathrm{S} 1413-81232018000100303$.

12. Filho EAF. Profile of alcohol and drug use in adolescent pupils in a Brazilian State capital. SMAD, Rev Eletrôn Saúde Mental Álcool Drog. [Internet]. 2014; 10(2):78-84. [cited Nov 17 2018]. Available from: http://pepsic.bvsalud.org/scielo.php?script=sci_ 
arttext\&pid=S1806-69762014000200005\&Ing=pt\&nrm =iso\&tlng=en

13. Facundo FRG, Pedrão LJ, Aguilar LR, Garcia KSL, Almanza SEE. Trastornos por consumo de alcohol (AUDIT) en adolescentes y jovenes marginales de bandas juveniles de Mexico. Esc Anna Nery. [Internet] 2007;11(4):611-8 [Acceso 17 nov 2018]. Disponible en:http://www.scielo.br/pdf/ean/v11n4/v11n4a09.pdf 14. Coutinho ESF, França-Santos D, Magliano ES, Bloch KV, Barufaldi LA, Cunha CF et al. ERICA: padrões de consumo de bebidas alcoólicas em adolescentes brasileiros. Rev Saúde Pública. [Internet] 2016; 50(supl 1):8s [Acesso 17 nov 2018]. Disponível em: http://www.scielo.br/pdf/rsp/v50s1/pt_0034-8910rsp-S01518-87872016050006684.pdf

15. Cardoso LRD, Malberguier A. Problemas escolares e o consumo de álcool e outras drogas entre adolescentes. Rev Quadrimestral Assoc Bras Psicol Escolar Educ. (São Paulo). [Internet] 2014;18(1):2734 [Acesso 17 nov 2018]. Disponivel em: http:// www.scielo.br/scielo.php?script $=$ sci_arttext\&pi $\mathrm{d}=\mathrm{S} 1413-85572014000100003$

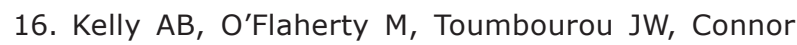
JP, Hemphill SA, Catalano RF. Gender differences in the impact of families on alcohol use: A lagged longitudinal study of early adolescents. Addiction. [Internet]. 2011; 106(8): 1427-36 [cited 2015 Nov 21]. Available from: https://www.researchgate.net/publication/50865441_ Gender_differences_in_the_impact_of_families_on_ alcohol_use_A_lagged_longitudinal_study_of_early_ adolescents

17. Filho EAF, Queiros PS, Medeiros M, Rosso CCW, Souza MM. Concepções sobre drogas por adolescentes escolares. Rev Bras Enferm. [Internet]. 2015;. 68(3):517-23. [Acesso 17 nov 2018]. Disponível em: http://www.scielo.br/scielo.php?script=sci_arttext\&pi $\mathrm{d}=$ S0034-71672015000300517

18. Arroyave LJO, Restrepo-Mendes MC, Horta BL, Menezes AMB, Gigante DP, Gonçalves H. Tendências e desigualdades nos comportamentos de risco em adolescentes: comparação das coortes de nascimentos de Pelotas, Rio Grande do Sul, Brasil. Cad Saúde Pública. [Internet]. 2016; 32(9):e00120215. [Acesso 17 nov 2018] Disponível em: http://www. scielo.br/scielo.php?script=sci_arttext\&pid=S0102311X2016000905013
19. Viero VSF, Farias JM, Ferraz F, Simões PW, Martins JA, Ceretta LB. Educação em saúde com adolescentes: análise da aquisição de conhecimentos sobre temas de saúde. Esc Anna Nery. [Internet] 2015;19(3):484-90. [Acesso 17 nov 2018]. Disponível em: http://www.scielo. br/pdf/ean/v19n3/1414-8145-ean-19-03-0484.pdf
Copyright $\odot 2019$ SMAD, Rev. Eletrônica Saúde Mental Álcool Drog. Este é um artigo de acesso aberto distribuído sob os termos da Licença Creative Commons CC BY-NC.

Esta licença permite que outros remixem, adaptem e criem a partir do seu trabalho para fins não comerciais, e embora os novos trabalhos tenham de lhe atribuir o devido crédito e não possam ser usados para fins comerciais, os usuários não têm de licenciar esses trabalhos derivados sob os mesmos termos. 